\title{
Caracterização dos acidentes de trânsito envolvendo trabalhadores motociclistas em Pernambuco - 2016
}

\section{Characterization of transit accidents involving motorcyclists in Pernambuco-2016}

\author{
Washington José dos Santos ${ }^{1}$ (D), Vanessa Maria da Silva Côelho² (BD , Gustavo Barreto Santos ${ }^{3}$ (D), Albanita Gomes da Costa de \\ Ceballos $^{4}$ (1)
}

1. Unidade de Terapia Intensiva do Hospital das Clínicas de Pernambuco da Universidade Federal de Pernambuco (UFPE), Recife, PE, Brasil. 2. Mestre em Saúde Coletiva pela Universidade Federal de Pernambuco (UFPE), Recife, PE, Brasil. 3. Docente da Faculdade Integrada de Vitória de Santo Antão (FAINTVISA), Recife, PE, Brasil. 4. Docente do Programa de Pós-graduação em Saúde Coletiva pela Universidade Federal de Pernambuco (UFPE), Recife, PE, Brasil.

\section{Resumo}

Introdução: os acidentes de trânsito configuram-se como problema de saúde pública devido à sua alta morbimortalidade. Embora haja poucos estudos sobre trabalhadores motociclistas, sabe-se que a maioria dos usuários de motocicletas vive em áreas urbanas e é formada por indivíduos que usam a moto para ir e voltar do trabalho. Objetivos: caracterizar os trabalhadores motociclistas que sofreram acidente de trânsito internados no Hospital da Restauração (HR) de Recife - PE e descrever as características do acidente sofrido por esses condutores. Métodos: estudo transversal realizado no leito de pacientes trabalhadores acidentados, condutores de motocicletas, internados no HR em Recife-PE, no período de maio a setembro de 2016, utilizando questionário com variáveis sociodemográficas, ocupacionais e relacionadas ao acidente. Resultados: foram entrevistados 124 indivíduos. A maioria era do sexo masculino (97,6\%), adulto jovem de 18 a 39 anos (81,5\%), com renda média mensal menor que 2 salários $(75,0 \%)$. Cerca de $55 \%$ dos entrevistados possuíam vínculo empregatício informal. 0 maior número de acidentes ocorreu entre 18:01 e 00:00 (45\%). Colisão com outros veículos automotores foi o tipo de acidente mais frequente $(62,0 \%)$. Conclusões: os resultados demonstram que a maioria dos acidentados era do sexo masculino, em idade produtiva, que ganhava menos de 2 salários mínimos e que a maioria dos acidentes se deu por colisão. A importância da educação e da sensibilização junto aos motociclistas e aos demais condutores, a fim de reduzir a incidência e a gravidade dos acidentes envolvendo motocicletas.

Palavras-chave: Acidente de trânsito. Motocicletas. Trabalhadores. Epidemiologia. Perfil de Saúde.

\begin{abstract}
Introduction: Traffic accidents are configured as public health problem due to their high morbidity and mortality. Although there are few studies on motorcycle workers, it is known that most motorcycle users live in urban areas and are made of individuals who use the bike to go to and from work. OBJECTIVES: To characterize motorcyclists who suffered a traffic accident hospitalized at a Recife Hospital - Recife, Brazil, and describe the characteristics of the accidents suffered by these drivers. Methods: A cross-sectional study carried out in the hospital bed of injured workers, drivers of motorcycles, hospitalized at the HR in Recife-PE, from May to September 2016, using a sociodemographic, occupational, and accident-related questionnaire. Results: 124 individuals were interviewed. The majority were males (97.6\%), young adults aged 18 to 39 years (81.5\%), with a monthly average income lower than 2 minimum wages (75.0\%). About 55\% of the interviewees had an informal employment relationship. The highest number of accidents occurred between 18:01 and 00:00 (45\%). Collision with other automobiles was the most frequent type of accident (62.0\%). Conclusions: The results showed that the majority of the accident victims were male, in a productive age, who earned less than 2 minimum wages and that most of the accidents occurred due to collision. The importance of education and awareness among motorcyclists and other drivers in order to reduce the incidence and severity of accidents involving motorcycles.
\end{abstract}

Key words: Traffic. Accidents. Motorcycles. Workers. Epidemiology. Health Profile

\section{INTRODUÇÃO}

Nos centros urbanos, a motocicleta tornou-se uma opção de transporte atrativa devido às dificuldades de acesso ao transporte público de qualidade, e também pelo baixo custo de aquisição, manutenção e facilidade de deslocamento em situações de tráfego intenso ${ }^{1,2}$.

No Estado de Pernambuco (PE), entre os anos de 2010 e 2015, a frota de motocicletas cresceu $78,46 \%{ }^{3}$ e, paralelamente, o estado passou a ocupar o décimo lugar no ranking do Brasil de mortes por acidentes de motocicletas com uma taxa de mortalidade de 9,6 por 100 mil habitantes. No Brasil, em 2016, o total de internações hospitalares no Sistema Único de Saúde devido a acidentes de transporte foi de 180.443, dessas 104.719 eram envolvendo acidentes com motociclistas. A Região Nordeste ficou em segundo lugar em internamentos de acidentes com motociclistas 34.292, e, desses, Pernambuco foi

Correspondência: Washington José dos Santos. Unidade de Terapia Intensiva do Hospital das Clínicas de Pernambuco da Universidade Federal de Pernambuco (UFPE), Iputinga, CEP: 50.670-901 - Recife, PE - Brasil. E-mail: washingtonfisio@gmail.com

Conflito de interesse: Não há conflito de interesse por parte de qualquer um dos autores.

Recebido em: 2 Maio 2018; Revisado em: 26 Jul 2018; 16 Ago 2018; Aceito em: 17 Ago 2018 
responsável por 3.587 registros ${ }^{4}$.

Os acidentes de trânsito configuram-se como um problema de saúde pública devido à sua alta morbimortalidade. As consequências das lesões dos ocupantes das motocicletas são maiores do que para os ocupantes de automóveis, podendo causar invalidez temporária ou permanente, trazendo um alto custo para o estado no cuidado dessas vítimas 5 .

Considerando o grande quantitativo de acidentes envolvendo motociclistas e o grande número de trabalhadores que usam a moto para se deslocar para o trabalho ou como instrumento de trabalho, esta pesquisa teve como objetivos caracterizar os trabalhadores motociclistas que sofreram acidente de trânsito internados no Hospital da Restauração (HR) de Recife - PE e descrever as características do acidente sofrido por esses condutores.

\section{MÉTODOS}

Esta pesquisa possui um desenho transversal descritivo. A população analisada foi composta pelo universo de trabalhadores maiores de 18 anos, acidentados no trânsito na condição de condutores de motocicleta e internados na enfermaria de traumato-ortopedia do HR. O hospital foi escolhido para a realização da pesquisa por ser referência no atendimento a traumas e unidade sentinela para acidentes com transporte terrestre no estado. O HR funciona com 723 leitos e são realizadas, em média, 2.200 internações mensais, principalmente nas áreas de traumatologia, clínica médica, clínica cirúrgica e neurologia ${ }^{6}$.

Foram considerados critérios de exclusão: pacientes com nível de consciência alterado que impedisse de responder ao questionário analisado por pontuação na escala de coma de Glasgow de moderado a grave, ou seja, valores iguais ou inferiores a 13 pontos e também pacientes que não estivessem em condições físicas de assinar o termo de consentimento ou que não fosse possível recolher a impressão digital no momento de aplicação dos questionários.

Para a coleta de dados, foi utilizado um questionário elaborado pelos pesquisadores e ajustado durante um projeto piloto de 15 dias. As variáveis sociodemográficas estudadas foram cor da pele autorreferida (branco, pardo, negro, outra/não sabe), sexo (masculino e feminino), idade, situação conjugal com companheiro (a) e sem companheiro (a), número de filhos, escolaridade (analfabeto, ensino fundamental incompleto/ completo, ensino médio completo/incompleto/ensino técnico, ensino superior completo/incompleto), renda mensal média (menor que 2 salários, 2 a 3 salários, 4 ou mais salários mínimos). As variáveis ocupacionais pesquisadas foram: ocupação principal, outra ocupação, tempo na ocupação principal, vínculo empregatício (formal e informal), contribuição previdenciária(sim e não), dias semanais trabalhados (até 5 dias e mais de cinco dias), horas semanais trabalhadas (até 8 horas diárias e mais de 8 horas diárias), descanso no trabalho (sim e não), tempo de descanso e local para descanso no trabalho (sim e não). Também foram analisadas variáveis relacionadas ao acidente: horário do acidente (00:01 às 6:00, 6:01 às 12:00, 12:01 às 18:00 e 18:01 às 00:00), natureza do acidente (colisão, queda e choque com objeto fixo), tipo de colisão (automóvel, motocicleta, bicicleta, pedestre e animal).

Não foi realizado cálculo de tamanho da amostra. O universo de acidentados que correspondia aos critérios do estudo foi convidado a participar da pesquisa. A coleta de dados foi diária durante 4 meses (maio a setembro de 2016). O banco de dados foi desenvolvido, alimentado e analisado descritivamente no programa Statistical Package for the Social Sciences (SPSS) versão 20.0.

A pesquisa foi conduzida dentro dos padrões exigidos pela Declaração de Helsinque e teve a aprovação dos Comitês de ética da Universidade Federal de Pernambuco e do Hospital da Restauração CAAE 53093116.0.0000.5208 e 53093116.0.3001.5198 respectivamente, conforme a Resolução no 466/12 do Conselho Nacional de Saúde do Brasil. Todos os indivíduos que concordaram em participar da pesquisa, assinaram o Termo de Consentimento Livre e Esclarecido (TCLE) em duas vias, ficando uma para o pesquisador e outra para o participante.

\section{RESULTADOS}

O total de pesquisados foi 124 indivíduos. Houve 12 recusas em participar da entrevista e 8 perdas. A média de idade foi de 31,44 anos (mediana $=29 ; d p= \pm 9,50$ ), sendo o mais novo com 18 e o mais velho com 64 anos. A maioria dos pesquisados era do sexo masculino (97,6\%), adulto-jovem de 18 a 39 anos (81,5\%), com 2 filhos ou menos $(84,7 \%)$, renda média mensal menor que 2 salários (75,0\%) (Tabela.1).

Tabela 1. Características sociodemográficas dos trabalhadores motociclistas internados no Hospital da Restauração - Recife/ PE, 2016.

\begin{tabular}{lrr}
\hline Variável & $\mathbf{N}$ & $\%$ \\
\hline Sexo & & \\
$\quad$ Masculino & 121 & 97,6 \\
$\quad$ Feminino & 3 & 2,4 \\
\hline Idade & & \\
18 a 29 anos & 63 & 50,8 \\
30 a 39 anos & 38 & 30,7 \\
40 e mais & 23 & 18,5 \\
Situação conjugal & & \\
Sem companheiro(a) & 61 & 49,2 \\
Com companheiro(a) & 63 & 50,8 \\
\hline
\end{tabular}




\begin{tabular}{|c|c|c|}
\hline Variável & $\mathbf{N}$ & $\%$ \\
\hline \multicolumn{3}{|l|}{ Número de filhos } \\
\hline 0 & 53 & 42,7 \\
\hline $1-2$ & 52 & 42,0 \\
\hline 3 ou mais & 19 & 15,3 \\
\hline \multicolumn{3}{|l|}{ Escolaridade } \\
\hline Analfabeto & 2 & 1,6 \\
\hline Ensino fundamental incompleto/completo & 51 & 41,1 \\
\hline $\begin{array}{l}\text { Ensino médio incompleto/completo/ensino } \\
\text { técnico }\end{array}$ & 58 & 46,6 \\
\hline Ensino superior incompleto/completo & 13 & 10,5 \\
\hline \multicolumn{3}{|l|}{ Cor da pele autorreferida } \\
\hline Branco & 25 & 20,2 \\
\hline Pardo & 57 & 46,0 \\
\hline Negro & 13 & 10,5 \\
\hline Outra/Não sabe & 29 & 23,4 \\
\hline \multicolumn{3}{|l|}{ Renda } \\
\hline Menor que 2 salários & 93 & 75,0 \\
\hline 2 a 3 salários & 26 & 21,0 \\
\hline 4 ou mais salários & 5 & 4,0 \\
\hline
\end{tabular}

Legenda: $\mathrm{N}$ = valor absoluto; \% = valor percentual.

No que se refere à ocupação principal da população estudada, as mais frequentes foram agricultor $(12,1 \%)$, comerciante $(11,3 \%)$ e motoboy/mototáxi (9,6\%). Do total de entrevistados, 39,4\% possuíam até 2 anos de profissão, 54,8\% dos entrevistados possuíam vínculo empregatício informal, 65,3\% trabalhavam até 8 horas diárias e $82,3 \%$ tinham horário de descanso do trabalho (Tabela 2).

Tabela 2. Características dos trabalhadores motociclistas internados no Hospital da Restauração - Recife/PE, 2016.

\begin{tabular}{lrr}
\hline Variável & N & \% \\
\hline Ocupação principal & 15 & 12,1 \\
Agricultor & 14 & 11,3 \\
Comerciante & 12 & 9,6 \\
Motoboy/mototaxista & 9 & 7,2 \\
Construção civil & 5 & 4,0 \\
Mecânico & 4 & 3,2 \\
Segurança/vigilante & 3 & 2,4 \\
Serviços Gerais & 62 & 50,2 \\
Outras & &
\end{tabular}

\section{Outra ocupação1}

Agricultor

310,0

\begin{tabular}{|c|c|c|}
\hline Variável & $\mathbf{N}$ & $\%$ \\
\hline Construção civil & 3 & 10,0 \\
\hline Mototáxi & 2 & 6,6 \\
\hline Eletricista & 2 & 6,6 \\
\hline Mecânico & 2 & 6,6 \\
\hline Outros & 18 & 60,2 \\
\hline \multicolumn{3}{|c|}{ Tempo na ocupação principal } \\
\hline 1 a 12 meses & 28 & 22,5 \\
\hline 13 a 24 meses & 21 & 16,9 \\
\hline 25 a 60 meses & 27 & 21,8 \\
\hline 61 a 120 meses & 25 & 20,1 \\
\hline 121 meses ou mais & 23 & 18,4 \\
\hline \multicolumn{3}{|l|}{ Vínculo empregatício } \\
\hline Formal & 56 & 45,2 \\
\hline Informal & 68 & 54,8 \\
\hline \multicolumn{3}{|c|}{ Contribuição Previdenciária² } \\
\hline Sim & 61 & 49,2 \\
\hline Não & 62 & 50,0 \\
\hline \multicolumn{3}{|c|}{ Dias trabalhados por semana ${ }^{3}$} \\
\hline Até 5 dias & 54 & 43,5 \\
\hline Mais de 5 dias & 65 & 52,4 \\
\hline \multicolumn{3}{|c|}{ Horas semanais trabalhadas 4} \\
\hline Até 8 horas & 81 & 65,3 \\
\hline Mais de 8 horas & 39 & 31,5 \\
\hline
\end{tabular}

\section{Descanso no trabalho5}

$\begin{array}{lll}\text { Sim } & 102 & 82,3 \\ \text { Não } & 21 & 16,9\end{array}$

\section{Tempo de descanso6}

Sem descanso

Até $30 \mathrm{~min}$

$31-60 \mathrm{~min}$

Mais de $60 \mathrm{~min}$

$43 \quad 35,5$

\section{Local para descanso7}

$\begin{array}{lll}\text { Sim } & 62 & 50,0 \\ \text { Não } & 61 & 49,2\end{array}$

Legenda: $\mathrm{N}=$ valor absoluto; $\%=$ valor percentual; ${ }^{1}$ Apenas os que exercem uma segunda ocupação $(\mathrm{N}=30)^{2,5,7} ; \mathrm{N}=123^{3} ; \mathrm{N}=119^{4} ; \mathrm{N}=120^{6}$; $\mathrm{N}=110$.

O maior número de acidentes ocorreu entre 18:01 e 00:00 (45\%), seguido do horário de $12: 01$ as $18: 00(27 \%)$. Colisão foi o tipo de acidente mais frequente na população estudada (72,5\%). As colisões aconteceram mais comumente com automóveis $(63,3 \%)$ ou com outra motocicleta $(27,8 \%)$ (Tabela 3$)$. 
Tabela 3. Características do acidente dos trabalhadores motociclistas internados no Hospital da Restauração - Recife/ PE, 2016.

\begin{tabular}{lll}
\hline Variável & $\mathbf{N}$ & $\mathbf{\%}$ \\
\hline Hora do acidente & & \\
00:01 às 6:00 & 13 & 10,5 \\
6:01 às 12:00 & 21 & 16,9 \\
12:01 às 18:00 & 34 & 27,4 \\
18:01 às 00:00 & 56 & 45,2
\end{tabular}

Natureza do acidente

\begin{tabular}{lrr} 
Colisão & 90 & 72,5 \\
Queda & 26 & 21,0 \\
Choque com objeto fixo & 8 & 6,5 \\
Tipos de colisão & & \\
Automóvel & 57 & 63,3 \\
Motocicleta & 25 & 27,8 \\
Bicicleta & 1 & 1,1 \\
Pedestre & 1 & 1,1 \\
Animal & 6 & 6,7 \\
\hline
\end{tabular}

Legenda: $\mathrm{N}$ = valor absoluto; \% = valor percentual.

\section{DISCUSSÃO}

A maioria dos acidentados era jovem do sexo masculino, recebia, no momento do estudo, renda menor que 2 salários, sofreu o acidente no início da noite até zero hora. Em relação ao acidente, predominou colisão, principalmente, com automóveis.

O perfil dos motociclistas acidentados desta pesquisa corrobora os achados da literatura no qual a predominância dos acidentados de motos era do sexo masculino $0^{5,7-11}$ e em pessoas que estavam em idade economicamente ativa, variando entre 18 e 39 anos $^{8,12-16}$.

A predominância de acidentes era do sexo masculino estando relacionada ao comportamento mais agressivo no trânsito, especialmente os jovens que, em motocicletas, fazem demonstrações de potência, de domínio do perigo, do desprezo pelas regras, situações que se revelam muito perigosas, expondo-os ao risco de acidente, o que se tem confirmado como fator de maior exposição do gênero no trânsito ${ }^{17}$.

O grande número de jovens e adultos-jovens acidentados encontrados na pesquisa, pode ser atribuído ao fato de que, ao começar a dirigir, eles passam a constituir população de alto risco, principalmente pela inexperiência na condução de veículos, além de outros fatores, como a impulsividade da idade atrelada ao maior consumo de álcool e outras drogas ${ }^{8,18}$.

Quanto à escolaridade, somados os analfabetos até o ensino fundamental completo, tem-se $42,7 \%$ dos entrevistados com baixa escolaridade, o que vem ao encontro de outros estudos $^{19,20,21,22}$. É possível que a baixa escolaridade esteja relacionada à falta de carteira de habilitação e, consequente, à imperícia para pilotar motocicletas.

Em relação à cor da pele, a cor parda foi referida pelos motociclistas. Quando somados os declarados negros e pardos, representam 56,5\% dos pesquisados. Esses resultados podem não exercer influência sobre os acidentes, uma vez que a composição étnica do Estado de Pernambuco é formada, entre outras, por $53,3 \%$ de pardos e $4,9 \%$ negros $^{23}$.

A maioria dos entrevistados recebia menos de 2 salários mínimos o que corrobora o estudo de Santos et al ${ }^{24}$. É presumível que o baixo custo de aquisição e manutenção da motocicleta, quando comparado ao automóvel, torne-a uma opção atraente e economicamente viável para a população com essa faixa de renda, tornando esses trabalhadores mais vulneráveis aos acidentes.

A ocupação mais frequentemente citada pelos pesquisados foi a de agricultor, o que difere com o estudo de Corgozinho e Montagner (2017), que encontrou, como profissão principal, motoboys no município de Ceilândia(DF) ${ }^{25}$. Segundo Santos et al. (2008), a moto tem sido muito utilizada nas propriedades rurais e, muitas vezes, sem registro, sem preparo e sem controle pelos órgãos de fiscalização ${ }^{24}$.

Mais da metade dos entrevistados possuía vínculo informal de trabalho e menos de 50\% referiram contribuir com a Previdência. Um dos principais fatores de preocupação decorrentes desse elevado grau de informali-dade no Brasil é o fato de que, quan $\neg$ do vítimas de acidentes de trabalho, esses trabalhadores não possuírem os benefícios da proteção concedida pela legislação trabalhista ${ }^{13}$, uma vez que, dependendo da gravidade do acidente e da necessidade de cirurgia, os acidentados de moto podem ficar mais de 6 meses sem retornarem a suas atividades laborais ${ }^{26}$.

Quanto à natureza do acidente, a maioria foi por colisão com automóvel, seguida de motocicletas. Achados semelhantes foram encontrados por Andrade et al. (2009); Kobayashi e Carvalho (2011); e Rocha e Schor (2013), cujos números de colisão chegaram a $69 \%, 74 \%$ e $84,7 \%$, respectivamente $e^{8,27,11}$.

Para Kobayashi e Carvalho (2011), as colisões entre motos e automóveis são comuns, devido ao intenso tráfego de veículos nos grandes centros urbanos e as lesões com motociclistas são mais graves por causa de sua alta exposição ${ }^{27}$. A cada 1,6 quilômetros percorridos, os motociclistas têm 34 vezes mais chances de morte em acidentes do que pessoas que dirigem outros veículos ${ }^{28}$

O horário de 18:01 e 00:00 foi o que mais ocorreu acidentes, seguido do intervalo de 12:01 a 18:00. Andrade et al. (2009) observaram resultados semelhantes entre $18-24 \mathrm{~h}$ ocorreram 
$38,3 \%$ e de $12-18 \mathrm{~h}$, o percentual foi de $31 \%$, primeiro e segundo percentuais respectivamente ${ }^{8}$.

Esse horário pode incluir o retorno para casa após o trabalho ou após momentos de lazer. De toda forma, são considerados fatores agravantes para os horários: as cobranças, o cansaço físico, as pressões acumuladas durante o dia, a ansiedade, as preocupações e até as influências climáticas ${ }^{29}$. Alta jornada de trabalho sem intervalo é considerada também como importante fator associado à maior ocorrência de acidentes no período noturno ${ }^{30}$.

Uma limitação do estudo é não ter pesquisado os casos de vítimas que foram atendidas na emergência do hospital, mas não foram internadas. Sendo só os casos internados, a pesquisa deixou de estudar as ocorrências menos graves, casos que tenham sido transferidos para outros serviços e casos que tiveram como desfecho o óbito. Outra limitação foi a realização do estudo em apenas um hospital. Mesmo o
HR, local do estudo, sendo referência em traumas do Estado de Pernambuco, concentrando a maioria dos atendimentos de urgências e emergências por causas externas, a restrição a um único serviço impossibilita fazer estimativas populacionais.

\section{CONCLUSÕES}

Os acidentes motociclísticos fazem parte do cotidiano das cidades. Os resultados da presente pesquisa demonstram a importância da educação e da conscientização junto aos motociclistas e demais condutores, a fim de reduzir a incidência e a gravidade dos acidentes envolvendo motocicletas, principalmente aos condutores do sexo masculino, em idade economicamente produtiva. Para tanto, é importante conhecer melhor essa realidade e esse perfil dos acidentados para discutir ações que possam focar no público-alvo, respeitando as suas características, no sentido de prevenir os acidentes, reduzir os danos à vida e economizar recursos públicos dos atendimentos assistenciais e previdenciários.

\section{REFERÊNCIAS}

1. Almeida GCM, Medeiros FCD, Pinto LO, Moura JMBO, Lima KC. Prevalence and factors associated with traffic accidents involving motorcycle taxis. Rev Bras Enferm [Internet]. 2016 ;69(2):359-65. doi: http://dx.doi.org/10.1590/00347167.2016690223i.

2. Golias ARC, Caetano R. Acidentes entre motocicletas: análise dos casos ocorridos no estado do Paraná entre julho de 2010 e junho de 2011. Cien Saude Colet. 2013 May; 18(5):1235-46. doi: http://dx.doi.org/10.1590/S141381232013000500008 .

3. Departamento Nacional de Trânsito. Frota de Veículos - 2015. [Internet]. Brasília: DENATRAN; 2016 [acesso 2016 Jul 29]. Disponível em: http://www. denatran.gov.br/frota2015.htm.

4. Ministério da Saúde[BR]. Departamento de Informática do SUS - DATASUS. Morbidade hospitalar do SUS por causas externas - por local de internação - Brasil. Internações por região. Grupo de Causas: V01 a V89. Período: 2015 [Internet]. Brasília: Ministério da Saúde; 2018 [acesso 2018 Jul 27] Disponível em: http://tabnet.datasus.gov.br/cgi/tabcgi.exe?sih/cnv/fruf.def.

5. Ganne N. Estudo sobre acidentes de trânsito envolvendo motocicletas na cidade de Corumbá e região, Estado do Mato Grosso do Sul, Brasil, no ano de 2007. RPAS. 2010;1(3):19-24. doi: 10.5123/S2176-62232010000300003.

6. Furtado BMASM. Araújo Junior JLC, Cavalcanti P. O perfil da emergência do Hospital da Restauração: uma análise dos possíveis impactos após a municipalização dos serviços de saúde. Rev bras epidemiol. 2004;7(3):279-89. doi: http://dx.doi.org/10.1590/S1415-790X2004000300006.

7. Souto CC, Reis FKW, Betolini RPT, Lins RSM, Souza SLB. Perfil das vítimas de acidentes de transporte terrestre relacionados ao trabalho em unidades de saúde sentinelas de Pernambuco, 2012-2014. Epidemiol. Serv. Saude. 2016; 25(2): 351-361. doi: http://dx.doi.org/10.5123/s1679-49742016000200014.

8. Andrade LM, Lima MA, Silva CHC, Caetano JA. Acidentes de motocicleta: características das vítimas e dos acidentes em hospital de Fortaleza - CE, Brasil. Rev Rene. 2009;10(4):52-9.

9. Seerig LM, Bacchieri G, Nascinmento GG, Barros AJD, Demarco FF. Use of motorcycle in Brazil: user profile, prevalence of use and traffic accidents occurrence - a population - based study. Ciênc. saude coletiva. 2016; 21(12): 3703-3710. doi: http://dx.doi.org/10.1590/1413-812320152112.28212015.

10. Abdul Manan MM, Jonsson T, Andras V. Development of a safety performance function for motorcycle accident fatalities on Malaysian primary roads. Saf sci. 2013 Dec;60:13-20.

11. Rocha GS, Schor N. Acidentes de motocicleta no município de Rio Branco: caracterização e tendências. Cien Saude Colet. 2013 Mar; 18(3):721-31. doi: http://dx.doi.org/10.1590/S1413-81232013000300018.

12. Jakobi HR, Cruz VA. Acidentes de trânsito em condutores de motocicletas e motonetas em Porto Velho no período de 2010 a 2014. Rev bras. med. trab.2017;15(1):54-62.

13. Amorim CR, Araújo EM de, Araújo TM de, Oliveira NF de. Acidentes de acidentes de trabalho com mototaxistas. Rev bras epidemiol. 2012;15(1):25-37. doi: http://dx.doi.org/10.1590/S1415-790X2012000100003.

14. Costa MJC, Mangueira JO. Perfil epidemiológico de ocorrências no trânsito no Brasil - revisão integrativa. S A N A R E. 2014 Jun-Dez; 13(2):110-6.

15. Rodrigues CL, Armond JE, Gorio C, Souza PC. Accidents involving motorcyclists and cyclists in the municipality of São Paulo: characterization and trends. Rev bras. ortop. 2014;49(6):602-6. doi: http://dx.doi.org/10.1016/j. rboe.2014.11.002.

16. Campos $\mathrm{Cl}$, Romão MNPV, Ferraz $A C P$, Raia AA Junior, Simões $A$. Hospitalizations by the Brazilian Health System due to traffic acidents. Procedia Soc Behav Sci. 2014 Dec; 162: 282-9.

17. Mendes R. Ansiedade nos motociclistas. Aná Psicológica. 2005; 23(1):43-7.

18. Caixeta CR, Minamisava R, Oliveira LMAC, Brasil VV. Morbidade por acidentes de transporte entre jovens de Goiânia, Goiás. Cien Saude Colet. 2009 Jul;14(5):1807-15. doi: http://dx.doi.org/10.1590/S1413-81232010000400021.

19. Biffe CRF, Harada A, Bacco AB, Coelho CS, Baccarelli JLF, Silva KL, et al. Perfil epidemiológico dos acidentes de trânsito em Marília, São Paulo,2012. Epidemiol. Serv. Saude. 2017, 26(2): 389-98. doi: http://dx.doi.org/10.5123/ s1679-49742017000200016.

20. Silva MGP, Silva VL, Vilela MRB, Gomes AOC, Falcão IV, Cabral AKPS, Lima, MLLT. Fatores associados às alterações fonoaudiológicas em vítimas de acidentes de motocicletas. CoDAS. 2016; 28(6): 745-52. doi: http://dx.doi. org/10.1590/2317-1782/20162015178.

21. Montenegro MMS, Duarte EC, Prado RR, Nascimento AF. Mortalidade de motociclistas acidentados de transporte no Distrito Federal, 1996 a 2007. Rev Saude Publica. 2011;45(3):529-38. doi: http://dx.doi.org/10.1590/S0034- 


\section{Acidentes de trânsito com trabalhadores motociclistas em Pernambuco}

\section{1}

22. Zabeu JLA, Zovico JRR, Pereira Júnior WN, Tucci Neto PF. Perfil de vítima de acidente motociclístico na emergência de um hospital universitário. Rev Bras Ortop. 2013;48(3):242-5. doi: https://doi.org/10.1016/j.rbo.2012.09.006.

23. Instituto Brasileiro de Geografia e Estatística. Estados: censo 2010 [Internet]. Rio de Janeiro: IBGE; 2011[acesso 2017 Jan 15] Disponível em http://www.ibge. gov.br/estadosat/perfil.php?sigla=pe.

24. Santos AMR, Moura MEB, Nunes BMVT, Leal CF dos S, Teles JBM. Perfil das vítimas de trauma por acidente de moto atendidas em um serviço público de emergência. Cad Saude Publica. 2008 Ago; 24(8):1927-1938. doi: http://dx.doi. org/10.1590/S0102-311X2008000800021.

25. Corgozinho MM, Montagner MA. Vulnerabilidade humana no contexto do trânsito motociclístico. Saúde soc. 2017; 26(2): 545-555. doi: 10.1590/S010412902017167713.

26. Fernandes FF, Reis CC, Câmara SMA, Maciel ACC. Fatores associados ao não retorno ao trabalho de indivíduos acidentados de moto: um estudo epidemiológico. RBSP. 2015; 39(2): 191-204.

27. Kobayashi CR, Carvalho MS. Violência urbana: acidentes de trânsito envolvendo motociclistas na cidade de Londrina (PR). Rev. Geografia (Londrina). 2011 Set-Dez; 20(3): 171-90. doi: http://dx.doi.org/10.5433/24471747.2011v20n3p171.

28. Lin M, Kraus JF. A review of risk factors and patterns of motorcycle injuries. Accid Anal Prev. 2009 Jul; 41(4):710-22. doi: 10.1016/j.aap.2009.03.010.

29. Albuquerque AM, Silva HCL, Torquato IMB, Gouveia BLA, Abrantes MSLA, Torres VSF. Vítimas de acidentes de moto com traumatismo. Rev enferm UFPE on line. 2016 Maio; 10(5): 1730-8. doi: 10.5205/reuol.9003-78704-1SM.1005201620.

30. Silva DW, Andrade SM, Soares DA, Nunes, EFPA, Melchior R. Condições de trabalho e riscos no trânsito urbano na ótica de trabalhadores motociclistas. Physis. 2008;18(2):339-60. doi: http://dx.doi.org/10.1590/S010373312008000200008 